\title{
Switching Between Biological Treatments in Psoriatic Arthritis: A Review of the Evidence
}

\author{
Luisa Costa $^{1} \cdot$ Carlo Perricone $^{2} \cdot$ Maria Sole Chimenti $^{3} \cdot$ Antonio Del Puente $^{1}$. \\ Paolo Caso $^{4} \cdot$ Rosario Peluso $^{1}$ Paolo Bottiglieri ${ }^{1} \cdot$ Raffaele Scarpa $^{1}$. \\ Francesco Caso 1
}

Published online: 20 October 2017

(c) The Author(s) 2017. This article is an open access publication

\begin{abstract}
Psoriatic arthritis (PsA) is a chronic inflammatory arthropathy. Therapy with anti-tumor necrosis factor (TNF)- $\alpha$ agents represents the first therapeutic choice for moderate and severe forms; however, PsA patients can experience anti-TNF $\alpha$ failure, lack of efficacy, or adverse events. Several evidences exist on the effectiveness of switching among different TNF $\alpha$ inhibitors, and we reviewed the published data on the effectiveness of antiTNF $\alpha$ first-, second- and third-line. Most of the studies report that the main reason for switching to a second anti$\mathrm{TNF} \alpha$ agent is represented by lack of efficacy (primary or secondary) and, more rarely, adverse events. Switchers receiving their second anti-TNF $\alpha$ agent have considerably poorer responses compared with non-switchers. Survival of anti-TNF $\alpha$ treatment appears to be superior in PsA patients when compared with rheumatoid arthritis patients. Switching from anti-TNF agents to ustekinumab or secukinumab or apremilast can represent a valid alternative therapeutic strategy.
\end{abstract}

Raffaele Scarpa

rscarpa@unina.it

1 Rheumatology Unit, Department of Clinical Medicine and Surgery, University Federico II, via S. Pansini 5, 80131 Naples, Italy

2 Rheumatology, Department of Internal Medicine, Sapienza University of Rome, Rome, Italy

3 Rheumatology, Allergology and Clinical Immunology, Department of "Medicina dei Sistemi", University of Rome "Tor Vergata", Rome, Italy

4 Geriatric Unit, Faculty of Medicine and Psychology, S. Andrea Hospital, "Sapienza" University of Rome, Rome, Italy

\section{Key Points}

Psoriatic arthritis (PsA) is a chronic inflammatory arthropathy associated with psoriasis, in which the recognition of different cytokines has changed the therapeutic approach.

Different studies have shown the efficacy and safety of anti-tumor necrosis factor (TNF)- $\alpha$ in PsA, but discontinuation or switching is quite common.

Data indicate that the main reason for switching to a second anti-TNF $\alpha$ agent is represented by lack of effect, followed by inefficacy and, more rarely, adverse events.

\section{Introduction}

Psoriatic arthritis (PsA) is a chronic inflammatory arthropathy associated with psoriasis in which ocular, intestinal, metabolic and cardiovascular involvement can variably occur, suggesting a comprehensive definition of the condition as psoriatic disease [1-6].

Dactylitis, entheseal and axial involvement associated with psoriasis or its familial history, as well as rheumatoid factor (RF) negativity, represent addressing diagnostic aspects [7, 8]. Ultrasonography (US) and magnetic resonance imaging (MRI) are useful tools for describing preradiological inflammatory phases and staging synovial and periarticular involvement [9-12].

The main targets of therapy are the achievement of clinical remission, improvement of patients' quality of life, and inhibition of structural radiological damage [13, 14]. 
Non-steroidal anti-inflammatory drugs (NSAIDs) and intra-articular corticosteroid injections are used prevalently in mild articular forms, and conventional synthetic diseasemodifying antirheumatic drugs (csDMARDs), including methotrexate, sulfasalazine, cyclosporine-A, and leflunomide, are required in more aggressive resistant cases [13, 14].

Biological DMARDs (bDMARDs) are recommended in severe cases, refractory to csDMARDs, possibly in the early phases of the disease. They are effective on inhibition of radiographic progression and on cutaneous and articular manifestations [13, 14].

Tumor necrosis factor (TNF)- $\alpha$ inhibition by use of its antagonists, represented by infliximab, etanercept, adalimumab, golimumab and certolizumab-pegol, has shown high efficacy in numerous randomized controlled trials (RCTs), and longitudinal and real-world studies [13-15]. Furthermore, despite the protective role of $\mathrm{TNF} \alpha$ against microorganisms, its inhibition has been shown to be cautiously safe if associated with appropriate screening and monitoring [16-20].

According to the progressive advances on the pathogenesis of PsA, other proinflammatory cytokines, such as interleukin (IL)-17/23, and enzymatic molecules, such as phosphodiesterase 4 (PDE4), have been recognized as key factors in PsA pathogenesis. This observation has opened up additional perspectives in the management of the disease with the use of the newly developed bDMARDS ustekinumab and secukinumab of the targeted synthetic DMARD (tsDMARD) apremilast [13, 21].

This situation has generated valid therapeutic strategies in cases of primary non-response, loss of efficacy with time, intolerance, side effects and contraindication to anti$\mathrm{TNF} \alpha$ agents $[22,23]$. Growing evidence suggests complex and variable treatment patterns for bDMARDs in PsA patients. Discontinuation or switching of biological agents due to tolerability issues or lack of efficacy is quite common, as is loss of efficacy over time.

In this review, we detail anti-TNFa failure, loss of efficacy, and withdrawal in PsA patients, and the treatment challenge that these patients subsequently face. The main published data on the effectiveness of second- and thirdline anti-TNFa (or restarting the index anti-TNFa treatment) will also be reported. We performed an extensive search of the PubMed electronic database using the following keywords: 'retention rate' or 'discontinuation rate' or 'switch' and 'psoriatic arthritis'.

\section{Data from Real-life Studies}

Several data describing the frequency of therapy switching and outcomes among PsA patients who switched anti$\mathrm{TNF} \alpha$ agent are derived from registries, including the
Danish nationwide DANBIO registry [24], Swedish Biologics Register (ARTIS) [25], British Society for Rheumatology Biologics (BSRB) Register [26, 27], South Swedish Arthritis Treatment Group (SSATG) register [28], and BIOBADASER (Spanish registry for adverse events of biological therapies in rheumatic disease) registry [29].

In detail, in the observational cohort study based on the DANBIO registry, during 10 years of follow up involving 1422 PsA patients starting an anti-TNFa agent, 548 patients $(39 \%)$ were reported as switching to a second TNF $\alpha$ inhibitor, mainly due to lack of efficacy, followed by adverse events [24]. Among these 548 patients, 245 were reported to continue treatment, whereas 189 switched to a third treatment and 114 patients stopped without starting other therapies. In the majority of switchers (62\%), the main cause for switching to a third biologic was represented by lack of efficacy. Among patients switching to a third treatment, $30 \%$ were reported as switching to a fourth biologic agent, and, among those, 35\% switched to a fifth anti-TNF $\alpha$ agent [24]. In this study, compared with baseline, a significant reduction of PsA activity after 3 and 6 months of therapy was reported during the first, second, and third anti-TNF $\alpha$ course [24].

Response rates were significantly lower with the second treatment when compared with the first anti-TNF $\alpha$ agent. Although response rates and drug survival were lower after switching, in a 2-year period an American College of Rheumatology 20\% (ACR20) response was reported in $47 \%$ of switchers after starting the first anti-TNF $\alpha$ agent. No differences were observed between anti-TNF $\alpha$ therapy combinations [24]. The study highlighted that more than half of the switchers were women and switchers showed a shorter disease duration (3 years) when compared with non-switchers (4 years) [24].

In a Swedish study examining 10 years of clinical experience (between 1999 and 2008), using the ARTIS register, several and remarkable temporal trends were found [25]. The study evaluated 1417 PsA patients, as well rheumatoid arthritis (RA) and spondyloarthritis (SpA) patients, starting a first biologic therapy, and showed that measures of disease activity at treatment start declined significantly for all diseases, and diminished between the start of first-, second- and third-line therapy [25]. In particular, in PsA patients, the median disease duration declined during the study period and no tendency on the use of any csDMARDs at biologic start was found; however, the rate of patients using corticosteroids and NSAIDs was lower in patients recently starting a bDMARD when compared with the 10-year biological initiators [25]. Up to one-third of all patients discontinued their first biologic treatment within 1 year of the start of therapy, during the period between 1999 and 2007. In this study, inefficacy and adverse events represented the main reasons for therapy 
discontinuation. Biological inefficacy was shown to increase with calendar time, and discontinuation led to a reduction in adverse events [25]. Switchers to biological agents showed different comorbidities. Despite a $<50 \%$ drug retention rate at 5 years, most patients remained exposed to some biologic [25].

Another study of 566 PsA patients receiving their first anti-TNF $\alpha$ agent, (etanercept, $n=316$; infliximab, $n=162$; adalimumab, $n=88$ ), which was included in the BSRB Register, was performed by Saad et al. in 2009, showing a high persistence rate in both initial and secondline anti-TNF $\alpha$-treated patients [26]. In this study, patients showed a mean age of $46 \pm 11$ years at baseline, 53\% were female, mean PsA duration was $12 \pm 9$ years, and persistence data were reported for a mean follow-up of $2.3 \pm 0.9$ person-years [26]. Results from this study showed that 422 patients completed at least 12 months of follow-up, $75.5 \%$ of whom remained on their first anti-TNF agent, while $9.5 \%$ discontinued for inefficacy, $10 \%$ for adverse events and 5\% for other reasons. The presence of comorbidities was shown to be associated with patients' withdrawal due to adverse events [26]. During the followup period, 178 patients received a second anti-TNF therapy and the survivor function on a second anti-TNF for switchers was $74 \%$ at 1 year [26].

In a subsequent study, data from the same registry showed that $>70 \%$ of PsA patients were responders at different times (6, 12 and 18 months) [27]. The response rate was comparable in patients receiving anti-TNF $\alpha$ agents in combination with a csDMARD or anti-TNF $\alpha$ monotherapy at 6 months. Disease remission was achieved by 27,36 and $35 \%$ of patients of the anti-TNF $\alpha$ cohort at 6 , 12 and 18 months, respectively [27].

In a 5-year period (April 1999-September 2006), data from the SSATG register evaluated potential predictors associated with the continuation of anti-TNF $\alpha$ therapies in 261 PsA patients starting anti-TNF therapy for the first time [28]. The main results of this study showed that response rates at 3-12 months for global VAS and pain were approximately 50\% [28]. Response rates were reported to be approximately $50 \%$. Initial use of etanercept $(p=0.01)$, high C-reactive protein (CRP) levels $(p=0.03)$ and concomitant therapy with methotrexate $(p=0.03)$ were associated with better overall drug survival, regardless of joint distribution involvement [28]. The improved drug survival of concomitant methotrexate was related to significantly fewer discontinuations related to adverse events. The rate of serious adverse events on antiTNF therapies was 5-6\% per year [28].

In 2006, in a study using the BIOBADASER registry, Gomez-Reino et al. reported that drug discontinuation was predicted by older age [29]. Of 4706 patients registered in the BIOBADASER registry between 2000 and 2004, 10\% were PsA patients. Survival was better in patients replacing the first TNF antagonist because of adverse events, and worse in patients older than 60 years of age or who were treated with infliximab [29]. Of the 289 PsA patients starting a first TNF inhibitor, 55 failed, and, in 15 patients starting a second agent, 8 failed. One- and 2-year drug survival rates of the TNF antagonist were 0.87 and 0.81 , respectively. Survival of the second TNF antagonist decreased to 0.68 and 0.60 at 1 and 2 years, respectively [29].

Among other studies focusing on a real-world setting, results from a hospital database of inflammatory arthropathies, including PsA, have shown that more than $30 \%$ of patients starting adalimumab, infliximab and etanercept discontinued their initial therapy, mainly due to primary (32\%) and secondary failure (37\%) and adverse events $(25 \%)$. Five-year retention on adalimumab $(77 \%)$ and etanercept (70\%) was higher than infliximab (17\%) [30].

In a clinical practice setting, the overall 10 -year retention rate of a first-line anti-TNF agent has been reported at approximately $23 \%$, while etanercept has shown a high drug survival rate. Results were significantly higher for SpA patients, including PsA, compared with RA patients [31].

Recently, Zhang et al. reported data from the Truven Health Analytics MarketScan ${ }^{\circledR}$ Research Database (2005-2009) on a total of 1698 and 3263 PsA patients who started a csDMARD and a bDMARD, respectively [32]. The study showed that PsA patients initiating a csDMARD or bDMARD did not remain on the first therapy for long [32]. Indeed, considering patients starting a csDMARD, more than $70 \%$ showed one or more therapy changes over the 1-year study period, with a median time of 85 days. After a therapy change, $83 \%$ discontinued, 29\% switched therapy (in the majority of cases switched to a bDMARD), and $25 \%$ received a therapy add-on mainly consisting of a bDMARD. For patients starting on a bDMARD, 46\% showed one or more therapy changes, with a median time of 110 days. Furthermore, among patients discontinuing therapy, $25 \%$ switched therapy (in more than $90 \%$ of cases switched to another bDMARD), and 7\% received a therapy add-on with a csDMARD [32].

Yeaw et al. performed a study evaluating treatment patterns within 360 days after discontinuation of anti-TNFa therapy, using the IMS LifeLink Health Plan Claims database (2005-2009) [33]. Among 27,704 patients showing different diagnoses of arthritis, including 2098 PsA patients who were anti-TNF $\alpha$ naive, 14,707 (53\%) patients discontinued treatment over 1-3 years of follow-up. Within 360 days of discontinuing the first anti-TNF $\alpha$ agent, 53\% of patients restarted the same therapy [33].

Of more than 2100 patients receiving anti-TNF $\alpha$ therapy (adalimumab, etanercept and infliximab), 52-64\% restarted 
the index agent, $17-21 \%$ switched to a non-index antiTNF $\alpha$ agent, $0.5-6 \%$ switched to a non-index biologic, $7 \%$ switched to non-biologic therapies, and 13-20\% did not receive any new treatment [33]. A significantly higher proportion of patients receiving etanercept restarted the same drug within 3 months of discontinuation, when compared with patients receiving adalimumab and infliximab [33].

Other studies analyzing treatment patterns among PsA patients who started biologic therapy were derived from the MarketScan ${ }^{\circledR}$ Commercial Claims and Encounters Database (Thomson Reuters, Ann Arbor, MI, USA) [34, 35].

In 2013, in a study from this database observing treatment patterns among 3164 PsA patients who started biologic therapy, csDMARDs combined with biologic agents were reported to reduce the rates of biologic therapy discontinuation [34]. Among PsA patients, 68\% started a biologic in monotherapy and 32\% started biologics combined with csDMARDs. In a 1 -year post-period, $51 \%$ of patients starting biologics continued the same treatment, with an average time to discontinuation of 279 days. Rates of discontinuation, switching, and restarting were 33, 10, and $6 \%$, respectively, for all patients. A significantly lower rate of discontinuation was observed in the biologic therapy combined with csDMARDs group compared with the biologic monotherapy group [34].

In 2012, using data from the MarketScan ${ }^{\circledR}$ database (2005-2009), Bonafede et al. analyzed etanercept, adalimumab, or infliximab treatment patterns within the first year after starting biological therapy in patients with different types of arthritis, including PsA [35]. The authors highlighted that, in the first year after initiating TNF blocker therapy, patients often have a more than 45-day treatment gap; however, approximately two-thirds of patients were either persistent with or restarted their index therapy in the year following TNF blocker initiation [35]. The authors found that among PsA patients, treatment patterns in the first year after starting etanercept, adalimumab, or infliximab were as follows: persistence ranging from 47 to $56 \%$; restarting the initial anti-TNF $\alpha$ agent after a treatment gap of more than 45 days $=29,17$, and $17 \%$ respectively; switching to a different biologic of interest, more than 10\%; and quitting ( $\geq 45$-day treatment gap with no restart or switch), ranging from 14 to $19 \%$. Thus, the combined rates of either persistence or restarting after a treatment gap among PsA patients were 76, 70, and $73 \%$ for etanercept, adalimumab, and infliximab, respectively [35]. Mean times for switching from the index anti-TNF $\alpha$ agent to one of the other two anti-TNF $\alpha$ agents ranged from 123 to 213 days [35].

In another study including 60 PsA patients (mean age 46 years, median disease duration 16 years) using biologic drugs (2001-2006), Coates et al. investigated the response to therapy and quantified non-response and outcome from switching agents [36]. The authors found that anti-TNFa therapy showed a sustained response over 3 years and was well tolerated [36]. The mean rate of improvement was found to be more than $50 \%$ in tender joint count (TJC), swollen joint count (SJC), and CRP levels, and $>35 \%$ in the overall PsA activity score. Improvements were sustained beyond 2 years with no loss of effect. Overall, in $90 \%$ of cases, a significant response was achieved, and switching was reported in $20 \%$ of cases. Outcomes were found to be similar regardless of the therapy used, PsA duration, and pattern [36]. The rate of non-response was low, with the majority of patients responding to secondand third-line therapies. Side effects leading to discontinuation or switching of first-line therapy were only found in $5 \%$ of patients, and non-response was reported in $20 \%$ long term [36].

Interesting findings on the reasons for medication discontinuation have been also described by the national survey data collected by the United States National Psoriasis Foundation using biannual surveys (2003-2011) [37]. In this study, conducted on 5604 patients with psoriasis and PsA, adverse effects, lack of effectiveness, and inadequate response represented the main causes of discontinuation of etanercept, adalimumab, infliximab, and golimumab [37]. Several data have reported that in case of inadequate response or adverse events with infliximab, early switching to etanercept therapy can be effective and safe [38].

An English regional survey was conducted on 548 PsA patients (median age 49 years; interquartile range [IQR] 40-57 years; $51 \%$ female) who started biologic therapy between August 2007 and June 2012 [39]. Median time from diagnosis to starting anti-TNF was 4.6 years (IQR $2.0-10.0$ years). At baseline, $72 \%$ of patients were receiving a concomitant csDMARD, and $84 \%$ of them were receiving methotrexate. The majority of patients were started on adalimumab first-line (64\%), followed by etanercept (34\%), infliximab (2\%) and golimumab (1\%) [39]. At the 12-week assessment, $74 \%$ of patients had an adequate response to anti-TNF $\alpha$ agents. The main reason for cessation of initial biologic and sequential use were secondary inefficacy and lack of efficacy over time. Among PsA patients receiving anti-TNF $\alpha$ therapy, $17 \%$ switched between anti-TNF $\alpha$ agents $(n=94)$, with a further $3 \%$ switching three to four biologics $(n=19)$ [39]. The majority of patients $(60 \%)$ had an adequate response to a second- or third-line biologic, with a further $18 \%$ of switchers awaiting assessment of their disease activity at the time of the survey [39].

In another routine care setting study conducted on 193 PsA patients (male/female, 107/86; mean age, 46.8 years; mean disease duration, 6.7 years, 171/22 peripheral/axial forms) who initiated anti-TNF $\alpha$ therapy between 2001 and 
2013 with a follow-up of at least 6 months, high drug survival and high response rates were observed [40]. The majority of patients started with first-line etanercept ( $n=102)$, followed by adalimumab $(n=46)$, infliximab $(n=44)$ and golimumab $(n=1)$. Only $25 \%$ of patients received concomitant csDMARDs $(65 \%$ receiving methotrexate) [40]. At 3 months, in $90 \%$ of patients an adequate response was achieved, while in 7 and 3\% of cases, discontinuation was observed due to lack of efficacy and adverse events, respectively. Median drug survival was 2 years, while 1- and 2-year drug survival rates were 77 and $67 \%$, respectively. Seventy-nine $(41 \%)$ patients switched to a second anti-TNFa, among whom $23 \%$ switched to a third anti-TNF $\alpha ; 82 \%$ of switchers responded to second-line therapy and $83 \%$ responded to third-line therapy [40].

Other data from a retrospective study, carried out in a routine clinical setting on 268 ankylosing spondylitis (AS) and PsA patients receiving a total of 353 anti-TNFa treatment courses (97 adalimumab, 180 etanercept, and 76 infliximab), showed that more than $70 \%$ of patients with a mean follow-up of 33.7 months were still receiving their initial therapy [41]. The majority of anti-TNF $\alpha$ treatment discontinuations were due to the absence of clinical remission $(53 \%)$ or the occurrence of an adverse event $(40 \%)$ [41]. The results of this study showed that, at a 6-month follow-up, more than $85 \%$ of patients who switched to another anti-TNF agent (due to the loss of efficacy over time or to the occurrence of an adverse event) were responsive to the second anti-TNF agent [41]. In this study, treatment courses were significantly shorter in women than in men, and axial or peripheral involvement did not seem to be determinant factors influencing the anti-TNF agent retention rate, which was only affected by therapy duration [41]. In particular, in the subset of patients with prevalent axial involvement, the authors found a longer duration of biologic therapy [41]. No significant differences were shown in the duration of anti-TNF therapy according to whether a patient was receiving a concomitant csDMARD or not [41].

The authors reported that comparison among different arthropathies showed significant differences between PsA and AS $(p=0.0073)$ and between PsA and PsA with predominant axial involvement $(p=0.0467)$ in terms of therapy duration, but not significant differences in persistence rate. Moreover, in the comparison among therapies, there was a significant difference relating to the treatment persistence rates between etanercept and infliximab $(p=0.0062)$. More than $85 \%$ of patients were still responding after 6 months of follow-up [41].

In a German retrospective cohort study conducted using the Electronic Medical Record database of IMS Disease Analyzer, Lyu et al. analyzed treatment persistence and treatment patterns of 197 PsA patients, 576 RA patients, and $108 \mathrm{AS}$ patients without biologic experience during the pre-index period, starting subcutaneous biologic therapies (from 2009 to 2012) with and without prior DMARD use [42]. The percentages of PsA-persistent patient$\mathrm{s} \geq 12$ months was $58 \%$, and the median persistence time over 12 months was 365.0 days (mean 264.1) [42]. Switching between subcutaneous biologics occurred in $<10 \%$ of patients in all three cohorts. In the subpopulations with at least two prescriptions for the index subcutaneous biologic, and who remained persistent on the index subcutaneous biologic, dose escalation of $\geq 50 \%$ occurred in more than $50 \%$ of PsA patients. In this study, a significant rate of patients who remained persistent on their index subcutaneous biologic had a dose escalation [42].

Another real-world setting, retrospective, observational analysis was performed using the Humana commercial claims database. This analysis evaluated patients with an index (first) claim (2008-2011) for biologic therapies for the treatment of different diseases, including psoriasis and PsA [53]. Among 2721 patients analyzed, 48\% were new patients and $52 \%$ were patients who were continuing their treatment. The percentage of patients with psoriasis, PsA, and associated psoriasis and PsA was 21.2, 6.3 and 7.6\%, respectively [43]. Continuing patients had higher rates of persistence on index therapy than patients receiving new treatment. New patients were less persistent than those continuing on therapy [43].

Most continuing patients were persistent on their index biologic at 1 year, and the rates of discontinuation from the index biologic were higher for new patients than for continuing patients [43]. The proportion of patients who restarted their index biologics after a 45-day gap in therapy varied by agent and indication, and, with some exceptions, appeared to be similar between new and continuing patients. Relatively few patients switched from their index biologics to another biologic. In particular, index biologic therapies and treatment patterns for each biologic over 360 days were analyzed for new and continuing patients [43].

In a 2014 multicenter, retrospective cohort study, Kádár et al. explored the main causes of permanent discontinuation of biological treatment in patients with different inflammatory arthritis, including 11 PsA patients in whom therapies had been permanently discontinued [44]. The most common causes of discontinuation were represented by adverse events, inefficacy, or remission [44].

In a 2013 study, Bonafede et al. examined, by way of a retrospective real-world analysis, etanercept and adalimumab treatment patterns in patients with psoriasis and PsA included in a US claims database [45, 46]. Among 4453 patients with psoriasis and PsA, 2534 and 1919 patients started etanercept and adalimumab, respectively. 
Treatment changes were common in all groups within the first 12 months of treatment with etanercept or adalimumab $[55,56]$. Among patients treated with etanercept, 46-61\% were persistent for $\geq 12$ months, $43-49 \%$ discontinued, 24-26\% restarted, and 5-21.4\% switched index therapy within 12 months $[45,46]$. Among patients treated with adalimumab, 57-63\% were persistent for $\geq 12$ months, 51-63\% discontinued, 12.6-22.4\% restarted, and 11-18\% switched index therapy within 12 months [45, 46].

In a retrospective study, Paccou et al. examined the data of $99 \mathrm{SpA}$ patients, including 21 PsA patients, who had received at least two anti-TNF $\alpha$ agents [47]. The authors showed that in case of failure or intolerance to anti-TNFa in the treatment of SpA, performing a first or second switch yields a satisfactory therapeutic response $(80.8 \%)$. After the failure of a third anti-TNF $\alpha$, a satisfactory response was rarely achieved (82.1\%) [47].

In a retrospective study investigating adalimumab as a second or third choice in 47 patients, including 25 PsA patients, adalimumab was shown to be a good second-line drug regardless of the reason for switching [48].

A retrospective study by the US Department of Veterans Affairs Medical Center pharmacy (from 1999-2007) evaluated the pattern of biologic agent use in 428 male patients with inflammatory diseases, including PsA and psoriasis, treated with biologics at some point over the 9 -year study period [49]. The mean number of biologics used was highest in patients with $\operatorname{SpA}(1.5, p=0.003)$, with the mean stop/switch rate for the first biologic agent being lowest in patients with RA $(47.4 \%, p=0.02)$. For all groups except inflammatory bowel disease, the biologic with the highest rate of continuation was etanercept [49].

Several prospective, observational, longitudinal studies have investigated the efficacy and drug discontinuation in PsA patients under biological therapies.

In a 5-year, open-label, observational cohort study evaluating 65 patients, Saougou et al. reported a sustained clinical response at the end of therapies, with a survival rate of $76 \%$ for etanercept, $57 \%$ for infliximab, and $50 \%$ for adalimumab. All anti-TNF agents were effective, safe, and well-tolerated. Clinical improvement (in terms of the Psoriatic Arthritis Response Criteria [PsARC], ACR50, Disease Activity Score [DAS]-28, and Psoriasis Area and Severity Index [PASI] 70 and 90) was maintained through the 5 years with high infliximab, adalimumab, and etanercept survival [50].

A prospective, observational study investigating 217 first-time PsA switchers or 57 second-time switchers between 2003 and 2012 showed that the response rates of first-time anti-TNF switchers were moderate, while the response rates of second-time switchers were poor [61]. In particular, the 3-month ACR20 Lund Efficacy Index (LUNDEX) response was achieved by $47 \%$ of first-time switchers and $22 \%$ of second-time switchers; ACR50 LUNDEX rates were 21 and 14\%, ACR70 LUNDEX rates were 12 and 2\%, and EULAR LUNDEX rates were 26 and $10 \%$, respectively. Median drug survival time for patients switching anti-TNF for the first time was 64 months (95\% confidence interval [CI] 31-97) compared with 14 months (95\% CI 5-23) for second-time switchers. The baseline predictor of ACR20 response to second-line treatment was the DAS-28 values at baseline (odds ratio [OR] 1.45, 95\% CI 1.01-2.10), while higher Health Assessment Questionnaire (HAQ) scores predicted premature drug withdrawal (hazard ratio [HR] 1.60, 95\% CI 1.03-2.48) [51].

A prospective, observational study investigating the possibility of drug-free remission in csDMARD- or bDMARD-treated PsA patients in continuous remission for at least 6 months showed that the hazard to reach drug-free remission in PsA patients was low [52]. In this study, the authors evaluated 14 patients receiving methotrexate monotherapy and 12 patients receiving anti-TNF therapy [52]. The incidence of recurrence of disease was high $(n=20,76.9 \%)$ and occurred rapidly $(74.50 \pm 51.72$ days $)$ after treatment discontinuation. Male PsA patients were significantly more likely to lose remission. Long disease duration, severe psoriasis, and the presence of synovial hypertrophy by ultrasonographic examination at baseline decreased the likelihood for drug-free remission. When restarting DMARDs, prompt remission followed in all cases [52].

A prospective study reported the 2-year drug survival rates of adalimumab, etanercept, and infliximab in 328 PsA patients with peripheral arthritis (213 with polyarticular PsA and 115 with oligoarticular PsA) beginning their first ever anti-TNF $\alpha$ treatment [53]. Persistence in therapy was not statistically different in the two subsets. In particular, 2-year drug survival rates were 65.7 and $70.4 \%$ in polyarticular and oligoarticular PsA, respectively. In the polyarticular subset, the persistence of etanercept $(68.3 \%)$ was significantly higher than that of adalimumab (51.9\%, $p=0.01$ ). No significant difference in oligoarticular PsA was shown. Female sex represented a predictor of drug discontinuation [53].

Important data on drug survival have been reported by longitudinal observational studies conducted in the context of the Norwegian longitudinal observational study on DMARDs (NOR-DMARD) [54-56]. Among these studies, in 2008, a comparison of the 1-year retention rates between 172, 847, and 249 anti-TNFa treatment courses in PsA, RA, and AS patients, respectively, showed that survival of anti-TNF treatment was superior in AS and PsA patients compared with RA patients [54]. Larger improvements in HRQOL in patients with PsA and AS may contribute to the differences in drug survival [54]. Unadjusted 1-year retention rates were 77.3, 77.5, and $65.4 \%$ in the PsA, AS 
and RA, groups, respectively, and the adjusted HR for treatment termination was 0.76 (95\% CI $0.53-1.07)$ for PsA versus RA. High baseline disease activity and female sex were significantly associated with premature treatment termination, whereas concomitant methotrexate was associated with better drug survival for PsA and RA patients, but not for AS patients [54].

In 2013, another longitudinal observational study in NOR-DMARD of 95 PsA patients starting their first antiTNF, and who had switched to a second agent, showed that $20-40 \%$ of patients had a response on a second anti-TNF [55]. In particular, switchers receiving their second antiTNF had significantly poorer responses compared with 344 non-switchers (ACR50 response: 22.5 vs. $40.0 \%$; DAS28 remission: 28.2 vs. $54.1 \%$ ). Within switchers, there was a trend toward poorer responses to the second anti-TNF compared with the first anti-TNF. Estimated 3-year drug survival was $36 \%$ for the second anti-TNF compared with $57 \%$ for the first anti-TNF [55].

In a successive analysis of data from NOR-DMARD, responses were similar in the two groups studied, including 170 patients starting their first anti-TNF as monotherapy and 270 patients with concomitant methotrexate. However, drug survival was superior in patients receiving methotrexate $(p=0.07)$, mainly for patients receiving infliximab $(p=0.01)$. In addition to the lack of concomitant methotrexate, current smoking was also found to be an independent predictor of discontinuation of biological therapies [56].

In a prospective, open-label, uncontrolled study investigating the efficacy, toxicity, and survival of infliximab in 32 PsA patients refractory to at least two DMARDs, infliximab was effective, safe, and well tolerated. Clinical response was maintained for a period of 3 years, with a high survival rate for infliximab [57]. In particular, eight patients withdrew from the study, primarily for acute allergic reactions. After the first year, the infliximab survival rate was 84 , and $75 \%$ after the second year, which was maintained throughout the third year of treatment [57].

With regard to infliximab, several studies suggested that a longer therapy period before discontinuation could be more effective in maintaining a good clinical response [58]. Indeed, in a 78-week, open-label study showing a sustained effect of infliximab at low dose $(3 \mathrm{mg} / \mathrm{kg}$ every 8 weeks) combined with methotrexate, the improvement obtained lasted a few months, even 1 year after infliximab discontinuation [58].

In a study by Conti et al. among patients who switched from infliximab to etanercept, a clinical response was demonstrated in $70 \%$ of PsA patients and $83 \%$ of AS patients [59].

In a study analyzing 301 patients with arthritis (46 patients with AS, 63 with PsA, and 192 with RA) treated with anti-
TNF agents, globally $38 \%$ of these patients received more than one anti-TNF agent, but anti-TNF switching in AS and PsA was less frequent than in RA patients. In particular, switching to a second anti-TNF $\alpha$ agent was seen in 115 (38\%) patients, namely 21 PsA, 11 AS, and 83 RA patients. PsA patients showed the best response rate to a second antiTNF therapy. Moreover, 46 patients ( 5 with AS, 3 with PsA and 38 with RA) received a third TNF blocker [60]. Survival of anti-TNF agents in AS and PsA seems to be better than in RA. Switching anti-TNF agents for loss of efficacy over time may have the best effect in patients with AS and PsA, and predominantly in RA [60].

Some authors suggest that switching due to lack of efficacy may be an alternative in AS and PsA patients. Furthermore, switching to a second anti-TNF agent as a result of side effects may be reasonable, while switching to a third anti-TNF agent, again as a result of side effects, cannot be recommended [60].

Successively, different studies on serum anti-drug antibodies have been conducted [61, 62].

In a Chinese study evaluating the effect of anti-drug antibodies on the clinical efficacy and withdrawal rate of anti-TNF $\alpha$ agents in patients with rheumatic diseases starting a first biologic, the production of anti-infliximab and anti-adalimumab, but not anti-etanercept, antibodies was shown as a key determinant of discontinuation of the related therapies [61]. In PsA and RA, the non-response rate was significantly higher in patients with anti-drug antibodies when compared with responders (54 vs. 13\%; $p=0.01)$ [61].

In a study evaluating 143 patients ( 62 with RA and 81 with SpA, among whom 49 had AS and 32 had PsA) treated with anti-TNF $\alpha$ agents for a median of 28 months, patients not responding to treatment had higher serum anti-adalimumab and anti-infliximab antibody levels. Anti-infliximab antibody formation was related to an increased risk of infusion reactions, changing to another biologic drug, and treatment discontinuation. Anti-etanercept antibodies were not found in any of the 61 etanercept-treated patients [62].

\section{Data from Randomized, Placebo-Controlled Trials}

In 2010, a study evaluating the effectiveness and safety of adalimumab $40 \mathrm{mg}$ biweekly showed that PsA patients previously treated with infliximab and/or etanercept obtained significant improvements after 12 weeks of therapy. In this study, patients were stratified by anti-TNF therapy history, previous anti-TNF therapy (infliximab discontinued for 2 or more months, etanercept discontinued for 3 or more weeks, or both), and causes of therapy discontinuation. At week 12 of adalimumab treatment, 
modified PsARC was fulfilled by $71 \%$ of 66 PsA patients with prior exposure to anti-TNFa agents, and by $79 \%$ of 376 patients with no previous infliximab or etanercept therapy. The percentages of PsA patients obtaining an improved Physician's Global Assessment of psoriasis increased from 33 to $61 \%$ for patients with prior treatment and from 35 to $70 \%$ for patients without previous use of anti-TNF therapy. Adalimumab was shown to be safe and adverse events were similar for patients with and without prior anti-TNF therapy [63].

Adalimumab has been reported to be safe, and also leads to major improvement with DMARDs [64].

Data on combined therapy of infliximab with methotrexate have been reported in a recent open-label study that evaluated the efficacy and safety of infliximab $(5 \mathrm{mg} / \mathrm{kg})$ at weeks 0,2 , 6 , and 14 combined with methotrexate (15 mg weekly) or methotrexate alone (15 mg weekly) in 115 PsA patients naive to methotrexate [65]. At week 16,86\% of patients receiving infliximab plus methotrexate, versus $67 \%$ of those receiving methotrexate alone, achieved a significantly greater improvement of ACR20 response rates, PASI 75, dactylitis, fatigue and duration of morning stiffness, CRP levels, DAS28 response, and remission rates. In the infliximab plus methotrexate group, two patients had serious adverse events, but none in the methotrexate group; however, combined therapy was generally well-tolerated [65].

In addition to the IMPACT study, other evidences on the efficacy of infliximab on radiological outcomes have also been reported by Marzo-Ortega et al. These authors evaluated 18 PsA patients receiving four infusions of infliximab (3 $\mathrm{mg} / \mathrm{kg}$ ) combined with methotrexate who underwent MRI of the inflamed hands (12 patients) or the knee joints (6 patients) before and after therapy. In addition to significant improvement in all clinical outcomes, combined therapy with infliximab and methotrexate was associated with an improvement in MRI bone edema [66].

In a recent phase III, randomized, placebo-controlled study (RAPID-PsA), Mease et al. reported on the efficacy and safety of certolizumab in PsA, showing results consistent with those of other anti-TNFa agents [67]. In this study, 409 patients were randomized $1: 1: 1$ to receive placebo, certolizumab $200 \mathrm{mg}$ every 2 weeks, or certolizumab $400 \mathrm{mg}$ every 4 weeks. Twenty percent of patients had been previously exposed to one anti-TNF $\alpha$ agent. Improvements in the signs and symptoms of PsA on certolizumab were observed at 1 week [67]. At week 12, ACR20 response showed a significant improvement $(p<0.001)$ in patients randomized to certolizumab $200 \mathrm{mg}$ every 2 weeks $(58.0 \%)$ and $400 \mathrm{mg}$ every 4 weeks $(52 \%)$ when compared with patients receiving placebo $(24.3 \%)$. Overall, 368 patients completed 24 weeks of treatment and certolizumab showed a safe profile. At week 24, skin involvement, enthesitis, dactylitis, nail disease and ACR20 response confirmed improvement in certolizumab- treated patients. The high 24-week ACR20 response in patients under certolizumab was independent of prior antiTNF $\alpha$ therapy. There was a statistically significant improvement in PsARC $(p<0.001)$ and physical function from baseline $(p<0.001)$ compared with the placebo group [67].

Significant improvement in the HAQ Disability Index (HAQ-DI), Short Form-36 (SF-36), Psoriatic Arthritis Quality of Life (PsAQOL), fatigue, pain, Dermatology Life Quality Index (DLQI), social participation, and productivity at paid work and within the home were observed in both certolizumab groups when compared with placebo [68, 69].

Post hoc analyses showed that certolizumab therapy was able to inhibit radiographic progression when compared with placebo, above all in patients with high baseline modified total sharp score (mTSS) and CRP concentrations [70]. In an extension study, certolizumab efficacy was maintained at week 96 in both dose regimens and in patients with/without prior anti-TNF exposure [71].

Recently, biosimilar drugs for anti-TNFa have been introduced due to the patent expiry of the originator molecule [72-74]. Several studies have investigated switching from the originator drug to a biosimilar molecule in PsA [75, 76]. Among those studies, NOR-SWITCH represents a randomized study showing that patients with inflammatory bowel disease, SpA, RA, PsA, or chronic plaque psoriasis receiving stable treatment with an infliximab originator for at least 6 months and switching from the originator molecule to a biosimilar anti-TNF $\alpha$ agent (Remsima, CT-P13) is not inferior to continued treatment with the originator drug [75].

Data from the DANBIO registry have reported that in 802 patients with arthritis, RA, PsA, and axial SpA treated with infliximab for a median of more than 6 years, switching to CT-P13 had no negative impact on 1-year clinical outcomes and disease activity [76].

\section{Switching from Anti-Tumor Necrosis Factor Agents to Ustekinumab, Secukinumab, Ixekizumab and Apremilast}

Among new therapies able to inhibit precise targets, ustekinumab, a fully human monoclonal antibody blocking the common p40 chain shared by IL-12 and IL-23 (anti-IL12p40), has also been recently approved in the US and Europe for adults patients with active PsA [77].

In four reports of two large phase III trials (PSUMMIT I and II), subcutaneous use of ustekinumab at a dose of 45 or $90 \mathrm{mg}$ showed greater effect than placebo on cutaneous, articular, and radiological aspects [78-81].

In the phase III, multicenter, placebo-controlled trial PSUMMIT II, which included 312 PsA patients with 
previous exposure to conventional and/or anti-TNF $\alpha$ agents, a significant and sustained improvement was shown in PsA patients receiving ustekinumab [80]. Patients were randomized to ustekinumab 45 or $90 \mathrm{mg}$ at week 0 , week 4 and every 12 weeks, or placebo at weeks 0 , 4, and 16, and crossover to ustekinumab $45 \mathrm{mg}$ at weeks 24, 28 and 40. At week 16, patients with a $<5 \%$ improvement in TJC/SJC entered blinded early escape (from placebo to ustekinumab $45 \mathrm{mg}$; from ustekinumab $45 \mathrm{mg}$ to ustekinumab $90 \mathrm{mg}$; from ustekinumab $90 \mathrm{mg}$ to ustekinumab $90 \mathrm{mg}$ ). Forty-four percent of patients receiving ustekinumab, compared with $20 \%$ of patients receiving placebo, achieved the primary endpoint, represented by ACR20 at week $24(p<0.001)$ [80]. Significant treatment differences were observed for the secondary endpoints, as determined by week 24 HAQ-DI improvement $(p<0.001), \quad$ ACR50 $\quad(p \leq 0.05)$, and PASI $75(p<0.001)$; these results were sustained through week 52. Among patients previously treated with one or more anti-TNF agent, sustained ustekinumab efficacy was also observed. Unexpected adverse events were not observed through week 60 [80].

Combined data from PSUMMIT I and II indicated that ustekinumab, regardless of dose of 45 or $90 \mathrm{mg}$, significantly inhibited radiographic progression at week 24 in PsA patients, as determined by total van der Heijde modified sharp score (vdH-S) score mean changes [81].

Secukinumab represents a fully human immunoglobulin (Ig) G1 $\kappa$ monoclonal antibody that selectively neutralizes IL-17A [77], which, acting with other proinflammatory cytokines, including $\mathrm{TNF} \alpha$, leads to upregulation of expression of different genes associated with inflammatory response in different cells, as well as keratinocytes and fibroblasts, leading to increased production of proinflammatory molecules [77].

Secukinumab was tested in two phase III, placebocontrolled RCTs, in both TNF inhibitor-naive and TNF inhibitor-experienced patients [82, 83]. The first, FUTURE-1, used secukinumab $10 \mathrm{mg} / \mathrm{kg}$ intravenously at weeks 0,2 , and 4 , followed by subcutaneous secukinumab at a dose of either 150 or $75 \mathrm{mg}$ every 4 weeks, or placebo [77]. Compared with placebo, significantly higher responses were observed for the two drug-treated groups in the achievement of ACR20. Secondary endpoints, including the ACR50 response and joint structural damage, were significantly better in the secukinumab groups than in the placebo group [82].

Successively, 397 patients with active PsA were included in a large, phase III, double-blind, placebo-controlled study. Patients were randomized to subcutaneously receive secukinumab $300 \mathrm{mg} \quad(n=100), 150 \mathrm{mg} \quad(n=100)$, $75 \mathrm{mg}(n=99)$, or placebo $(n=98)$ once weekly from baseline and then every 4 weeks from week 4 . The results showed that subcutaneous secukinumab 300 and $150 \mathrm{mg}$ improved the signs and symptoms of PsA [83].

In the SPIRIT-P2 phase III trial, ixekizumab, another monoclonal antibody that inhibits IL-17A, at a dosage of $80 \mathrm{mg}$ every 4 weeks or every 2 weeks after a $160 \mathrm{mg}$ starting dose, has been shown to be efficacious in improving the signs and symptoms of patients with active PsA who previously had inadequate response to anti-TNFa agents [84].

Another trial on ixekizumab conducted in biologic-naive PsA patients has reported efficacy in improving disease activity and physical function, as well as in the inhibition of structural damage progression. In regard to safety, adverse events have been reported more frequently in all active groups compared with placebo [85].

Apremilast is an oral molecule that inhibits PDE4, a key intracellular hydrolysing cyclic adenosine monophosphate (cAMP) into AMP [77]. The clinical efficacy and safety of apremilast has been reported in the Psoriatic Arthritis Long-term Assessment of Clinical Efficacy (PALACE) phase III pivotal clinical trials (PALACE 1, 2, and 3), which demonstrate that apremilast is effective and safe for the treatment of active PsA, despite prior conventional DMARD or biologic treatment [86-88].

\section{Discussion}

Data on therapy in case of failure of biologic agents are still scarce in PsA and derive from registries [24-29], prospective and longitudinal studies [50-62], and randomized, placebo-controlled trials [63-71]. Most of the evidence indicates that the main reason for switching to a second anti-TNF $\alpha$ agent is represented by lack of effect, followed by inefficacy and, more rarely, adverse events [24-26, 30, 41]. Switchers receiving their second antiTNF $\alpha$ agent have considerably poorer responses compared with non-switchers [55]. Drug survival for the second anti$\mathrm{TNF} \alpha$ agent has been found to be lower when compared with drug-survival for the first anti-TNF $\alpha$ agent overall [55]. In the majority of cases, the main cause for switching to a third biologic relies on the lack of effect [24].

A sustained clinical response has been reported at 5 years of observation, with satisfying infliximab and adalimumab survival rates and high etanercept survival [50]. No differences between the various anti-TNF $\alpha$ combinations have been observed to date [24]. Survival seems to be better in patients replacing the first TNF antagonist as a result of adverse events [29].

In most cases, patients who switched to another antiTNF agent due to the loss of efficacy over time or to the occurrence of an adverse event seem to be more responsive to the second therapy [41]. Survival of patients receiving 
anti-TNF $\alpha$ treatment appears to be superior in PsA patients compared with RA patients [54, 60].

In several studies, axial or peripheral involvement did not seem to be a determinant factor in influencing the antiTNF agent retention rate, only the duration of therapy [41]. Additionally, persistence in therapy has also been found to be similar in polyarticular and oligoarticular PsA subsets beginning their first anti-TNFa treatment [53]. The presence of other comorbidities has been reported to be frequently associated with patients' withdrawal due to adverse events [26].

On the other hand, the majority of switchers were women, and switchers showed a shorter disease duration, higher PsA severity, and mean duration when compared with non-switchers [24-26]. In particular, female sex has been found to be a predictor of drug discontinuation [53]. Furthermore, current smoking was also found to be an independent predictor of discontinuation of biological therapies [56].

It has been reported that therapy with etanercept, concomitant use of methotrexate, and high CRP levels at the start of therapy are potential predictors associated with the continuation of anti-TNF therapies and overall drug survival [28]. In particular, etanercept has been reported as the most persistent anti-TNF drug, with high survival rates $[31,53]$. For patients starting on a bDMARD, a lower number showed one or more therapy changes compared with patients starting on a csDMARD [32]. CsDMARDs combined with biologic agents were shown to reduce the rates of biologic therapy discontinuation [34].

In a recent systematic review by Behrens et al. evaluating evidence on the efficacy and safety of anti-TNFo monotherapy versus add-on methotrexate treatment, it was confirmed that the use of concomitant methotrexate was well tolerated and able to prolong drug survival of TNF inhibitors; however, no significant improvement with combined therapy was found [89]. The authors suggested that a possible therapeutic strategy in PsA patients with inadequate response to methotrexate could be to add an anti-TNF $\alpha$ agent. In case of a good response, methotrexate could be tapered and then possibly withdrawn, or, alternatively, initiation of TNF inhibitor monotherapy could be followed by the addition of methotrexate if a partial response is verified [89].

Drug survival has been shown to be greater in patients receiving methotrexate, mainly patients receiving infliximab [54, 56]. With regard to infliximab, several studies have suggested that after its discontinuation, a longer therapy period before discontinuation could be more effective for maintaining a good clinical response [58]. Drug discontinuation has also been reported to be predicted by older age, in patients older than 60 years, or patients who were treated with infliximab [29]. Treatment courses were significantly shorter in women than in men [41]. Among patients who switched from infliximab to etanercept, a clinical response has been shown in a high percentage of patients [59].

Several studies have highlighted that in PsA patients who started biologics, the production of anti-infliximab and anti-adalimumab, but not anti-etanercept, represents a key determinant for the discontinuation of related therapies $[61,62]$.

Recently, certolizumab efficacy has been reported at week 96 with both dose regimens and in patients with prior anti-TNF exposure [71].

Different studies have shown that switching from one anti-TNF $\alpha$ agent to another as a result of lack of efficacy may be an alternative in PsA patients. Switching to a second anti-TNF agent as a result of side effects may be reasonable, however switching to a third anti-TNF agent as a result of side effects cannot be recommended [60]. In clinical practice, switching from one anti-TNF $\alpha$ to another has been reported to be a successful strategy in patients who did not response to a previous anti-TNFa [24, 26, 38]. Moreover, in patients with radiographic progression, switching to another biological drug, or combined treatment with a csDMARD, can be considered [28]; however, evidence of increased efficacy of biologic therapy and patient survival by using csDMARDs remains to be more deeply investigated [28].

Studies investigating switching from the originator antiTNF $\alpha$ agent to a biosimilar molecule in PsA have reported no negative impact on disease activity, but, to date, data are still scarce [75, 76].

In a chart review study, Wolf et al. assessed, in clinical practice, how clinical outcomes may be influenced by switching or discontinuing anti-TNF therapies when not clinically necessary. PsA switchers, and patients who discontinued for non-medical, economic reasons showed a significant worsening of outcomes and increased disease flares when compared with patients who continued taking their anti-TNF agent [90].

The Italian board for the TAilored BIOlogic therapy (ITABIO) has recently reviewed the most consistent studies to indicate the best strategy for second-line biologic choice in patients with PsA [91]. The results of the study showed that patients' preference, the indication for antiTNF $\alpha$ monotherapy in fertile women who were potentially childbearing, and the intravenous route associated with dose titration in obese patients were valid [91]. Moreover, the authors suggested that, in patients not responding to the first anti-TNF, the strategy to switch to a second agent is suitable, considering the evidence of adalimumab efficacy in patients showing concomitant uveitis. In addition, the severity of psoriasis, of articular involvement, and the predominance of enthesitis and/or dactylitis may determine 
a patient's choice of either ustekinumab or secukinumab in PsA [91].

A recent observational study based on data from the Corrona registry of 520 PsA patients (190 receiving antiTNFa monotherapy, 217 receiving methotrexate monotherapy, and 113 receiving combined treatment) reported that physicians are unlikely to initiate biologic monotherapy [92].

With regard to switching to another mode of action, data are limited to secukinumab, ustekinumab and ixekizumab trials. In these studies, response to the drug was considered, both in patients naive to anti-TNF agents and those who had not responded to such treatment. All trials confirm their efficacy, both in TNF-naive patients and those previously treated [80, 83, 84].

In particular, switching from anti-TNF agents to ustekinumab, secukinumab and apremilast can be considered a valid alternative therapeutic strategy in patients with previous exposure to anti-TNF $\alpha$ agents [81, 82, 86].

In a recent review by Merola et al., PsA treatment recommendations from different groups, rheumatology societies, and organizations were evaluated for identifying consensus guidelines on switching between bDMARDs. The results of this study showed that switching bDMARD therapies is a recommended strategy for patients who experience treatment failure [93]. The authors highlighted that switching between anti-TNF $\alpha$ agents can be effective for many patients, but bDMARDs with different mechanisms of action may be a better therapeutic strategy alternative [92].

Rheumatology is a field that is constantly progressing, and pathogenetic aspects, as well as the clinical spectrum of inflammatory manifestations, represent domains still to be fully clarified [94, 95]. Due to peculiar PsA pathogenetic and clinical aspects, further studies on cytokines other than $\mathrm{TNF} \alpha$, as well as studies on molecular pathways, could elucidate treatment patterns [96-98].

In case of adverse events related to biologic agents, or inefficacy or lack of efficacy, no specific biological agent has been demonstrated to be more effective than others. The decision to switch to another drug should mainly be made according to the drug's safety profile, its comorbidities, previous therapy, and the patients' preferences (e.g. administration time and route). Evidence suggests complex and variable treatment patterns for bDMARDs in PsA patients. Discontinuation or switching of biological agents due to tolerability issues or lack of efficacy is quite common, as is loss of efficacy over time.

Although most of the available evidence is related to the effectiveness of switching among the approved TNF inhibitors, emerging RCT data on ustekinumab and apremilast in patients with prior anti-TNF exposure seem to be promising.

\section{Compliance with Ethical Standards}

Conflict of interest Luisa Costa, Carlo Perricone, Maria Sole Chimenti, Antonio Del Puente, Paolo Caso, Rosario Peluso, Paolo Bottiglieri, Raffaele Scarpa, and Francesco Caso declare no conflicts of interest.

Funding/financial support The authors have no funding to report.

Open Access This article is distributed under the terms of the Creative Commons Attribution-NonCommercial 4.0 International License (http://creativecommons.org/licenses/by-nc/4.0/), which permits any noncommercial use, distribution, and reproduction in any medium, provided you give appropriate credit to the original author(s) and the source, provide a link to the Creative Commons license, and indicate if changes were made.

\section{References}

1. Moll JM, Wright V. Psoriatic arthritis. Semin Arthritis Rheum. 1973;3:55-78.

2. Egeberg A, Khalid U, Gislason GH, et al. Association of psoriatic disease with uveitis: a Danish Nationwide Cohort Study. JAMA Dermatol. 2015;151(11):1200-5.

3. Scarpa R, Manguso F, D'Arienzo A, et al. Microscopic inflammatory changes in colon of patients with both active psoriasis and psoriatic arthritis without bowel symptoms. J Rheumatol. 2000;27:1241-6.

4. Costa L, Caso F, Atteno M, et al. Impact of 24-month treatment with etanercept, adalimumab, or methotrexate on metabolic syndrome components in a cohort of 210 psoriatic arthritis patients. Clin Rheumatol. 2014;33:833-9.

5. Costa L, Caso F, Ramonda R, et al. Metabolic syndrome and its relationship with the achievement of minimal disease activity state in psoriatic arthritis patients: an observational study. Immunol Res. 2015;61:147-53.

6. Costa L, Caso F, D'Elia L, et al. Psoriatic arthritis is associated with increased arterial stiffness in the absence of known cardiovascular risk factors: a case control study. Clin Rheumatol. 2012;31:711-5.

7. Caso F, Costa L, Atteno M, et al. Simple clinical indicators for early psoriatic arthritis detection. Springerplus. 2014;3:759.

8. Marchesoni A, Atzeni F, Spadaro A, et al. Identification of the clinical features distinguishing psoriatic arthritis and fibromyalgia. J Rheumatol. 2012;39:849-55.

9. Fiocco U, Stramare R, Coran A, et al. Vascular perfusion kinetics by contrast-enhanced ultrasound are related to synovial microvascularity in the joints of psoriatic arthritis. Clin Rheumatol. 2015;34:1903-12.

10. Soscia E, Sirignano C, Catalano O, et al. New developments in magnetic resonance imaging of the nail unit. J Rheumatol Suppl. 2012;89:49-53.

11. Fiocco U, Sfriso P, Oliviero F, et al. Blockade of intra-articular TNF in peripheral spondyloarthritis: its relevance to clinical scores, quantitative imaging and synovial fluid and synovial tissue biomarkers. Jt Bone Spine. 2013;80:165-70.

12. Scarpa R, Caso F, Costa L, et al. Psoriatic disease: clinical staging. J Rheumatol Suppl. 2015;93:24-6.

13. Coates LC, Kavanaugh A, Mease PJ, Soriano ER, Laura AcostaFelquer M, Armstrong AW, et al. Group for research and assessment of psoriasis and psoriatic arthritis 2015 treatment recommendations for psoriatic arthritis. Arthritis Rheumatol. 2016;68:1060-71. 
14. Gossec L, Smolen JS, Ramiro S, de Wit M, Cutolo M, Dougados $\mathrm{M}$, et al. European League Against Rheumatism (EULAR) recommendations for the management of psoriatic arthritis with pharmacological therapies: 2015 update. Ann Rheum Dis. 2016;75:499-510.

15. Caso F, Lubrano E, Puente AD, et al. Progress in understanding and utilizing TNF- $\alpha$ inhibition for the treatment of psoriatic arthritis. Expert Rev Clin Immunol. 2016;12(3):315-31.

16. Caso F, Cantarini L, Morisco F, et al. Current evidence in the field of the management with TNF- $\alpha$ inhibitors in psoriatic arthritis and concomitant hepatitis $\mathrm{C}$ virus infection. Expert Opin Biol Ther. 2015;15:641-50.

17. Atteno M, Costa L, Matarese A, et al. The use of TNF- $\alpha$ blockers in psoriatic arthritis patients with latent tuberculosis infection. Clin Rheumatol. 2014;33:543-7.

18. Costa L, Caso F, Atteno M, et al. Long-term safety of anti-TNF- $\alpha$ in PsA patients with concomitant HCV infection: a retrospective observational multicenter study on 15 patients. Clin Rheumatol. 2014;33:273-6.

19. Sanduzzi A, Bocchino M, Atteno M, et al. Screening and monitoring of latent tubercular infection in patients taking tumor necrosis factor- $\alpha$ blockers for psoriatic arthritis. J Rheumatol Suppl. 2012;89:82-5.

20. Costa L, Caso F, Del Puente A, Di Minno MN, Peluso R, Scarpa R. Incidence of malignancies in a cohort of psoriatic arthritis patients taking traditional disease modifying antirheumatic drug and tumor necrosis factor inhibitor therapy: an observational study. J Rheumatol. 2016;43:2149-54.

21. Scarpa R, Costa L, Atteno M, et al. Psoriatic arthritis: advances in pharmacotherapy based on molecular target. Expert Opin Pharmacother. 2013;14:2311-3.

22. Caso F, Costa L, Del Puente A, Scarpa R. Psoriatic arthritis and TNF inhibitors: advances on effectiveness and toxicity. Expert Opin Biol Ther. 2015;15:1-2.

23. Atzeni F, Costa L, Caso F, et al. Role of agents other than tumor necrosis factor blockers in the treatment of psoriatic arthritis. J Rheumatol Suppl. 2015;93:79-81.

24. Glintborg B, Ostergaard M, Krogh NS, Andersen MD, Tarp U, Loft AG, et al. Clinical response, drug survival, and predictors thereof among 548 patients with psoriatic arthritis who switched tumor necrosis factor $\alpha$ inhibitor therapy: results from the Danish Nationwide DANBIO Registry. Arthritis Rheum. 2013;65:1213-23.

25. Simard JF, Arkema EV, Sundström A, Geborek P, Saxne T, Baecklund E, et al. Ten years with biologics: to whom do data on effectiveness and safety apply? Rheumatology (Oxford). 2011;50:204-13.

26. Saad AA, Ashcroft DM, Watson KD, Hyrich KL, Noyce PR, Symmons DP, British Society for Rheumatology Biologics Register. Persistence with anti-tumour necrosis factor therapies in patients with psoriatic arthritis: observational study from the British Society of Rheumatology Biologics Register. Arthritis Res Ther. 2009;11:R52.

27. Saad AA, Ashcroft DM, Watson KD, Symmons DP, Noyce PR, Hyrich KL, British Society for Rheumatology Biologics Register. Efficacy and safety of anti-TNF therapies in psoriatic arthritis: an observational study from the British Society for Rheumatology Biologics Register. Rheumatology (Oxford). 2010;49:697-705.

28. Kristensen LE, Gulfe A, Saxne T, Geborek P. Efficacy and tolerability of anti-tumour necrosis factor therapy in psoriatic arthritis patients: results from the South Swedish Arthritis Treatment Group register. Ann Rheum Dis. 2008;67:364-9.

29. Gomez-Reino JJ, Carmona L, BIOBADASER Group. Switching TNF antagonists in patients with chronic arthritis: an observational study of 488 patients over a four-year period. Arthritis Res Ther. 2006;8:R29.
30. Ip K, Hartley L, Solanki K, White D. Retention on anti-tumour necrosis factor therapy: the Waikato experience. N Z Med J. 2015;128:34-40.

31. Biggioggero M, Favalli EG. Ten-year drug survival of anti-TNF agents in the treatment of inflammatory arthritides. Drug Dev Res. 2014;75(Suppl 1):S38-41.

32. Zhang HF, Gauthier G, Hiscock R, Curtis JR. Treatment patterns in psoriatic arthritis patients newly initiated on oral nonbiologic or biologic disease-modifying antirheumatic drugs. Arthritis Res Ther. 2014;16:420.

33. Yeaw J, Watson C, Fox KM, Schabert VF, Goodman S, Gandra SR. Treatment patterns following discontinuation of adalimumab, etanercept, and infliximab in a US managed care sample. Adv Ther. 2014;31:410-25.

34. Zhu B, Edson-Heredia E, Gatz JL, Guo J, Shuler CL. Treatment patterns and health care costs for patients with psoriatic arthritis on biologic therapy: a retrospective cohort study. Clin Ther. 2013;35:1376-85.

35. Bonafede M, Fox KM, Watson C, Princic N, Gandra SR. Treatment patterns in the first year after initiating tumor necrosis factor blockers in real-world settings. Adv Ther. 2012;29:664-74.

36. Coates LC, Cawkwell LS, Ng NW, Bennett AN, Bryer DJ, Fraser $\mathrm{AD}$, et al. Sustained response to long-term biologics and switching in psoriatic arthritis: results from real life experience. Ann Rheum Dis. 2008;67:717-9.

37. Armstrong AW, Robertson AD, Wu J, Schupp C, Lebwohl MG. Undertreatment, treatment trends, and treatment dissatisfaction among patients with psoriasis and psoriatic arthritis in the United States: findings from the National Psoriasis Foundation surveys, 2003-2011. JAMA Dermatol. 2013;149:1180-5.

38. Delaunay C, Farrenq V, Marini-Portugal A, Cohen JD, Chevalier $\mathrm{X}$, Claudepierre $\mathrm{P}$. Infliximab to etanercept switch in patients with spondyloarthropathies and psoriatic arthritis: preliminary data. J Rheumatol. 2005;32:2183-5.

39. Jani M, Macphie E, Rao C, Moore S, Mirjafari H, McLoughlin Y, et al. Effectiveness of switching between biologics in psoriatic arthritis- results of a large regional survey. Clin Med (Lond). 2014;14:95-6.

40. Soubrier AS, Bele-Philippe P, Cortet B, Ramdane-Sebbane N, Bacle-Boutry MA, Lemeunier L, et al. Treatment response, drug survival and safety of anti-tumour necrosis factor $\alpha$ therapy in 193 patients with psoriatic arthritis: a twelve-year "real life" experience. Jt Bone Spine. 2015;82:31-7.

41. Fabbroni M, Cantarini L, Caso F, Costa L, Pagano VA, Frediani $\mathrm{B}$, et al. Drug retention rates and treatment discontinuation among anti-TNF- $\alpha$ agents in psoriatic arthritis and ankylosing spondylitis in clinical practice. Mediat Inflamm. 2014;2014:862969.

42. Lyu R, Govoni M, Ding Q, Black CM, Kachroo S, Fan T, et al. Treatment persistence among patients with rheumatoid disease (RA, AS, PsA) treated with subcutaneous biologics in Germany. Rheumatol Int. 2016;36:143-53.

43. Howe A, Eyck LT, Dufour R, Shah N, Harrison DJ. Treatment patterns and annualdrug costs of biologic therapies across indications from the Humana commercial database. J Manag Care Spec Pharm. 2014;20:1236-44.

44. Kádár G, Balázs E, Soós B, Laduver A, Keszthelyi P, Szekanecz $\mathrm{Z}$, et al. Disease activity after the discontinuation of biological therapy in inflammatory rheumatic diseases. Clin Rheumatol. 2014;33:329-33.

45. Bonafede M, Johnson BH, Fox KM, Watson C, Gandra SR. Treatment patterns with etanercept and adalimumab for psoriatic diseases in a real-world setting. J Dermatolog Treat. 2013;24:369-73. 
46. Chastek B, Fox KM, Watson C, Gandra SR. Etanercept and adalimumab treatment patterns in psoriatic arthritis patients enrolled in a commercial health plan. Adv Ther. 2012;29:691-7.

47. Paccou J, Solau-Gervais E, Houvenagel E, Salleron J, Luraschi H, Philippe $\mathrm{P}$, et al. Efficacy in current practice of switching between anti-tumour necrosis factor- $\alpha$ agents in spondyloarthropathies. Rheumatology (Oxford). 2011;50:714-20.

48. Spadaro A, Punzi L, Marchesoni A, Lubrano E, Mathieu A, Cantini $\mathrm{F}$, et al. Switching from infliximab or etanercept to adalimumab in resistant or intolerant patients with spondyloarthritis: a 4-year study. Rheumatology (Oxford). 2010;49:1107-11.

49. Tran S, Hooker RS, Cipher DJ, Reimold A. Patterns of biologic agent use in older males with inflammatory diseases: an institution-focused, observational post-marketing study. Drugs Aging. 2009;26:607-15.

50. Saougou I, Markatseli TE, Papagoras C, Voulgari PV, Alamanos $\mathrm{Y}$, Drosos AA. Sustained clinical response in psoriatic arthritis patients treated with anti-TNF agents: a 5-year open-label observational cohort study. Semin Arthritis Rheum. 2011;40:398-406.

51. Kristensen LE, Lie E, Jacobsson LT, Christensen R, Mease PJ, Bliddal $\mathrm{H}$, et al. Effectiveness and feasibility associated with switching to a second or third tnf inhibitor in patients with psoriatic arthritis: a cohort study from Southern Sweden. J Rheumatol. 2016;43:81-7.

52. Araujo EG, Finzel S, Englbrecht M, Schreiber DA, Faustini F, Hueber A, et al. High incidence of disease recurrence after discontinuation of disease-modifying antirheumatic drug treatment in patients with psoriatic arthritis in remission. Ann Rheum Dis. 2015;74:655-60.

53. Iannone F, Lopriore S, Bucci R, Scioscia C, Anelli MG, Notarnicola A, et al. Two-year survival rates of anti-TNF- $\alpha$ therapy in psoriatic arthritis (PsA) patients with either polyarticular or oligoarticular PsA. Scand J Rheumatol. 2015;44:192-9.

54. Heiberg MS, Koldingsnes W, Mikkelsen K, Rødevand E, Kaufmann C, Mowinckel P, et al. The comparative one-year performance of anti-tumor necrosis factor alpha drugs in patients with rheumatoid arthritis, psoriatic arthritis, and ankylosing spondylitis: results from a longitudinal, observational, multicenter study. Arthritis Rheum. 2008;59:234-40.

55. Fagerli KM, Lie E, van der Heijde D, Heiberg MS, Kalstad S, Rødevand E, et al. Switching between TNF inhibitors in psoriatic arthritis: data from the NOR-DMARD study. Ann Rheum Dis. 2013;72:1840-4.

56. Fagerli KM, Lie E, van der Heijde D, Heiberg MS, Lexberg AS, Rødevand $\mathrm{E}$, et al. The role of methotrexate co-medication in TNF-inhibitor treatment in patients with psoriatic arthritis: results from 440 patients included in the NOR-DMARD study. Ann Rheum Dis. 2014;73:132-7.

57. Voulgari PV, Venetsanopoulou AI, Exarchou SA, Alamanos Y, Tsifetaki N, Drosos AA. Sustained clinical response and high infliximab survival in psoriatic arthritis patients: a 3-year longterm study. Semin Arthritis Rheum. 2008;37:293-8.

58. Covelli M, Scioscia C, Iannone F, Lapadula G. Repeated infusions of low-dose infliximab plus methotrexate in psoriatic arthritis: immediate benefits are not maintained after discontinuation of infliximab. Clin Exp Rheumatol. 2005;23:145-51.

59. Conti F, Ceccarelli F, Marocchi E, Magrini L, Spinelli FR, Spadaro A, et al. Switching tumour necrosis factor alpha antagonists in patients with ankylosing spondylitis and psoriatic arthritis: an observational study over a 5-year period. Ann Rheum Dis. 2007;66:1393-7.

60. Haberhauer G, Strehblow C, Fasching P. Observational study of switching anti-TNF agents in ankylosing spondylitis and psoriatic arthritis versus rheumatoid arthritis. Wien Med Wochenschr. 2010;160:220-4.

61. Mok CC, van der Kleij D, Wolbink GJ. Drug levels, anti-drug antibodies, and clinical efficacy of the anti-TNF $\alpha$ biologics in rheumatic diseases. Clin Rheumatol. 2013;32:1429-35.

62. Arstikyte I, Kapleryte G, Butrimiene I, Venalis A. Influence of immunogenicity on the efficacy of long-term treatment with TNF $\alpha$ blockers in rheumatoid arthritis and spondyloarthritis patients. Biomed Res Int. 2015;2015:604872.

63. Rudwaleit M, Van den Bosch F, Kron M, Kary S, Kupper H. Effectiveness and safety of adalimumab in patients with ankylosing spondylitis or psoriatic arthritis and history of anti-tumor necrosis factor therapy. Arthritis Res Ther. 2010;12:R117.

64. Karanikolas GN, Koukli EM, Katsalira A, Arida A, Petrou D, Komninou E, et al. Adalimumab or cyclosporine as monotherapy and in combination in severe psoriatic arthritis: results from a prospective 12-month nonrandomized unblinded clinical trial. J Rheumatol. 2011;38:2466-74.

65. Baranauskaite A, Raffayová H, Kungurov NV, Kubanova A, Venalis A, Helmle L, RESPOND investigators, et al. Infliximab plus methotrexate is superior to methotrexate alone in the treatment of psoriatic arthritis in methotrexate-naive patients: the RESPOND study. Ann Rheum Dis. 2012;71:541-8.

66. Marzo-Ortega H, McGonagle D, Rhodes LA, Tan AL, Conaghan PG, O'Connor P, et al. Efficacy of infliximab on MRI-determined bone oedema in psoriatic arthritis. Ann Rheum Dis. 2007;66:778-81.

67. Mease PJ, Fleischmann R, Deodhar AA, Wollenhaupt J, Khraishi $\mathrm{M}$, Kielar D, et al. Effect of certolizumab pegol on signs and symptoms in patients with psoriatic arthritis: 24-week results of a Phase 3 double-blind randomised placebo-controlled study (RAPID-PsA). Ann Rheum Dis. 2014;73:48-55.

68. Gladman D, Fleischmann R, Coteur G, Woltering F, Mease PJ. Effect of certolizumab pegol on multiple facets of psoriatic arthritis as reported by patients: 24-week patient-reported outcome results of a phase III, multicenter study. Arthritis Care Res (Hoboken). 2014;66:1085-92.

69. Kavanaugh A, Gladman D, van der Heijde D, Purcaru O, Mease $P$. Improvements in productivity at paid work and within the household, and increased participation in daily activities after 24 weeks of certolizumab pegol treatment of patients with psoriatic arthritis: results of a phase 3 double-blind randomised placebo-controlled study. Ann Rheum Dis. 2015;74:44-51.

70. van der Heijde D, Fleischmann R, Wollenhaupt J, Deodhar A, Kielar D, Woltering F, et al. Effect of different imputation approaches on the evaluation of radiographic progression in patients with psoriatic arthritis: results of the RAPID-PsA 24-week phase III double-blind randomised placebo-controlled study of certolizumab pegol. Ann Rheum Dis. 2014;73:233-7.

71. Mease P, Deodhar A, Fleischmann R, Wollenhaupt J, Gladman $\mathrm{D}$, Leszczyński $\mathrm{P}$, et al. Effect of certolizumab pegol over 96 weeks in patients with psoriatic arthritis with and without prior antitumour necrosis factor exposure. RMD Open. 2015;1:e000119.

72. Braun J, Kudrin A. Switching to biosimilar infliximab (CT-P13): Evidence of clinical safety, effectiveness and impact on public health. Biologicals. 2016;44(4):257-66.

73. Switching from innovator to biosimilar (subsequent entry) infliximab: an updated review of the clinical effectiveness, costeffectiveness, and guidelines. Ottawa (ON): Canadian Agency for Drugs and Technologies in Health; 2017. Available at http:// www.ncbi.nlm.nih.gov/books/NBK442045/.

74. Faccin F, Tebbey P, Alexander E, Wang X, Cui L, Albuquerque $T$. The design of clinical trials to support the switching and alternation of biosimilars. Expert Opin Biol Ther. 2016;16(12):1445-53. 
75. Jørgensen KK, Olsen IC, Goll GL, NOR-SWITCH study group, et al. Switching from originator infliximab to biosimilar CT-P13 compared with maintained treatment with originator infliximab (NOR-SWITCH): a 52-week, randomised, double-blind, non-inferiority trial. Lancet. 2017;389:2304-16.

76. Glintborg B, Sørensen IJ, Loft AG, All departments of rheumatology in Denmark, et al. A nationwide non-medical switch from originator infliximab to biosimilar CT-P13 in 802 patients with inflammatory arthritis: 1-year clinical outcomes from the DANBIO registry. Ann Rheum Dis. 2017;76:1426-31.

77. Caso F, Del Puente A, Peluso R, Caso P, Girolimetto N, Del Puente A, et al. Emerging drugs for psoriatic arthritis. Expert Opin Emerg Drugs. 2016;21(1):69-79.

78. McInnes IB, Kavanaugh A, Gottlieb AB, Puig L, Rahman P, Ritchlin C, PSUMMIT 1 Study Group, et al. Efficacy and safety of ustekinumab in patients with active psoriatic arthritis: 1 year results of the phase 3, multicentre, double-blind, placebo-controlled PSUMMIT 1 trial. Lancet. 2013;382:780-9.

79. Kavanaugh A, Puig L, Gottlieb AB, Ritchlin C, Li S, Wang Y, PSUMMIT I Study Group, et al. Maintenance of clinical efficacy and radiographic benefit through 2 years of ustekinumab therapy in patients with active psoriatic arthritis: results from the PSUMMIT 1 trial. Arthritis Care Res (Hoboken). 2015;67(12):1739-49.

80. Ritchlin C, Rahman P, Kavanaugh A, McInnes IB, Puig L, Li S, PSUMMIT 2 Study Group, et al. Efficacy and safety of the antiIL-12/23 p40 monoclonal antibody, ustekinumab, in patients with active psoriatic arthritis despite conventional non-biological and biological anti-tumour necrosis factor therapy: 6-month and 1-year results of the phase 3, multicentre, double-blind, placebocontrolled, randomised PSUMMIT 2 trial. Ann Rheum Dis. 2014;73:990-9.

81. Kavanaugh A, Ritchlin C, Rahman P, Puig L, Gottlieb AB, Li S, PSUMMIT-1 and 2 Study Groups, et al. Ustekinumab, an anti-IL12/23 p40 monoclonal antibody, inhibits radiographic progression in patients with active psoriatic arthritis: results of an integrated analysis of radiographic data from the phase 3, multicentre, randomised, double-blind, placebo-controlled PSUMMIT-1 and PSUMMIT-2 trials. Ann Rheum Dis. 2014;73:1000-6.

82. Mease PJ, McInnes IB, Kirkham B, et al. Secukinumab inhibition of interleukin-17A in patients with psoriatic arthritis. N Engl J Med. 2015;373(14):1329-39.

83. McInnes IB, Mease PJ, Kirkham B, et al. Secukinumab, a human anti-interleukin-17A monoclonal antibody, in patients with psoriatic arthritis (FUTURE 2): a randomised, double-blind, placebocontrolled, phase 3 trial. Lancet. 2015;386(9999):1137-46.

84. Nash P, Kirkham B, Okada M, SPIRIT-P2 Study Group, et al. Ixekizumab for the treatment of patients with active psoriatic arthritis and an inadequate response to tumour necrosis factor inhibitors: results from the 24-week randomised, double-blind, placebo-controlled period of the SPIRIT-P2 phase 3 trial. Lancet. 2017;389(10086):2317-27.

85. Mease PJ, van der Heijde D, Ritchlin CT, SPIRIT-P1 Study Group, et al. Ixekizumab, an interleukin-17A specific monoclonal antibody, for the treatment of biologic-naïve patients with active psoriatic arthritis: results from the 24-week randomised, doubleblind, placebo-controlled and active (adalimumab)-controlled period of the phase III trial SPIRIT-P1. Ann Rheum Dis. 2017;76(1):79-87.

86. Cutolo M, Myerson GE, Fleischmann RM, Lioté F, Díaz-González F, Van den Bosch F, et al. A phase III, randomized, controlled trial of apremilast in patients with psoriatic arthritis: results of the PALACE 2 trial. J Rheumatol. 2016;43(9):1724-34.

87. Kavanaugh A, Mease PJ, Gomez-Reino JJ, Adebajo AO, Wollenhaupt J, Gladman DD, et al. Treatment of psoriatic arthritis in a phase 3 randomised, placebo-controlled trial with apremilast, an oral phosphodiesterase 4 inhibitor. Ann Rheum Dis. 2014;73(6):1020-6.

88. Edwards CJ, Blanco FJ, Crowley J, Birbara CA, Jaworski J, Aelion J, et al. Apremilast, an oral phosphodiesterase 4 inhibitor, in patients with psoriatic arthritis and current skin involvement: a phase III, randomised, controlled trial (PALACE 3). Ann Rheum Dis. 2016;75(6): 1065-73.

89. Behrens F, Cañete JD, Olivieri I, van Kuijk AW, McHugh N, Combe B. Tumour necrosis factor inhibitor monotherapy vs combination with MTX in the treatment of PsA: a systematic review of the literature. Rheumatology (Oxford). 2015;54:915-26.

90. Wolf D, Skup M, Yang H, et al. Clinical outcomes associated with switching or discontinuation from Anti-TNF inhibitors for nonmedical reasons. Clin Ther. 2017;39(4):849-862.e6.

91. Cantini F, Niccoli L, Nannini C, et al. Second-line biologic therapy optimization in rheumatoid arthritis, psoriatic arthritis, and ankylosing spondylitis. Semin Arthritis Rheum. 2017;47(2):183-92.

92. Mease PJ, Lesperance T, Liu M, et al. Changes in treatment patterns in patients with psoriatic arthritis initiating biologic and nonbiologic therapy in a clinical registry. J Rheumatol. 2017;44(2):184-92.

93. Merola JF, Lockshin B, Mody EA. Switching biologics in the treatment of psoriatic arthritis. Semin Arthritis Rheum. 2017;47(1):29-37.

94. Caso F, Rigante D, Vitale A, Lucherini OM, Costa L, Atteno M, et al. Monogenic autoinflammatory syndromes: state of the art on genetic, clinical, and therapeutic issues. Int $\mathrm{J}$ Rheumatol. 2013;2013:513782.

95. Caso F, Costa L, Rigante D, Lucherini OM, Caso P, Bascherini $\mathrm{V}$, et al. Biological treatments in Behçet's disease: beyond antiTNF therapy. Mediat Inflamm. 2014;2014:107421.

96. Fiocco U, Accordi B, Martini V, Oliviero F, Facco M, Cabrelle A, et al. JAK/STAT/PKC $\delta$ molecular pathways in synovial fluid $\mathrm{T}$ lymphocytes reflect the in vivo $\mathrm{T}$ helper-17 expansion in psoriatic arthritis. Immunol Res. 2014;58:61-9.

97. Fiocco U, Martini V, Accordi B, Caso F, Costa L, Oliviero F, et al. Transcriptional network profile on synovial fluid $\mathrm{T}$ cells in psoriatic arthritis. Clin Rheumatol. 2015;34:1571-80.

98. Fiocco U, Martini V, Accordi B, Caso F, Costa L, Oliviero F, et al. Ex vivo signaling protein mapping in $\mathrm{T}$ lymphocytes in the psoriatic arthritis joints. J Rheumatol Suppl. 2015;93:48-52. 